\title{
Influence of cholecystectomy on sphincter of Oddi motility
}

\author{
W Luman, A J K Williams, A Pryde, G D Smith, S J Nixon, R C Heading, K R Palmer
}

\begin{abstract}
Background-Gall bladder and sphincter of Oddi (SO) function are coordinated by hormonal and neuronal mechanisms. Nerve fibres pass between the gall bladder and the SO via the cystic duct. It is therefore possible that cholecystectomy may alter SO motility.

Aim-To investigate the effect of cholecystectomy on SO function

Methods-SO manometry was performed in five women (median age 52 years), a few days before and six months after laparoscopic cholecystectomy which was undertaken for uncomplicated cholelithiasis. Basal and post-cholecystokinin (CCK) SO motility were measured.

Results-All patients were symptom free after laparoscopic cholecystectomy. Prior to surgery common bile duct pressure, and tonic and phasic SO motility were normal and phasic contractions were inhibited by intravenous CCK (1 Ivy Dog Unit/kg). Six months later, common bile duct pressure and baseline tonic and phasic activity were unchanged but CCK failed to suppress phasic activity. Conclusion-Cholecystectomy, at least in the short term, suppresses the normal inhibitory effect of pharmacological doses of CCK on the SO. The mechanism of this effect is unknown but it could be due to $S O$ denervation.
\end{abstract}

(Gut 1997; 41: 371-374)

Keywords: sphincter of Oddi; cholecystectomy; cholecystokinin

Gastrointestinal Unit, Western General Hospital, Crewe Road, Edinburgh EH4 2XU, Scotland, UK W Luman

A J K Williams

G D Smith

S J Nixon

K R Palmer

Centre for Digestive and Liver Disease, Royal Infirmary

Edinburgh, Scotland

A Pryde

R C Heading

Correspondence to:

Dr K R Palmer.

Accepted for publication 25 March 1997

Ingestion of a fatty meal is followed by release of cholecystokinin (CCK) which causes the gall bladder to contract and the sphincter of Oddi (SO) to relax. Coordination of gall bladder and SO function may also be influenced by nerve bundles which connect the gall bladder and SO via the cystic duct. ${ }^{1-3}$ Cholecystectomy may influence normal SO function by disrupting this nerve pathway and altering its response to $\mathrm{CCK}$.

To investigate the effect of cholecystectomy on SO function, biliary manometry was serially undertaken in a group of patients undergoing laparoscopic cholecystectomy for uncomplicated cholelithiasis.

Subjects and Methods

PATIENTS

Five female patients (aged 32-61, median 52 years) awaiting laparoscopic cholecystectomy for symptomatic cholelithiasis were studied. None had choledocholithiasis as judged by normal serum liver function tests and normal biliary tree on ultrasound scan. None had undergone gastric surgery or vagotomy, or had diabetes, significant cardiovascular, or respiratory disease. In addition, no patient was taking drugs which affect gastrointestinal motility. All patients completed a symptom questionnaire which detailed aspects of their presenting history, other abdominal symptoms, and medication.

The study was approved by the Lothian Ethics Subcommittee for Medicine and Oncology. Each patient was fully informed of the potential complications of biliary manometry and that the studies were not therapeutic.

\section{BILIARY MANOMETRY}

Biliary manometry was performed by a single operator (KRP) two to three weeks before and six months after laparoscopic cholecystectomy. After an overnight fast, patients were sedated with 5-10 mg intravenous midazolam. A standard triple lumen polyethylene manometric catheter (SOM-21-LEHMAN, Wilson Cook Medical Inc., Winston-Salem, USA) with an external diameter of $1.7 \mathrm{~mm}$, luminal diameter of $0.5 \mathrm{~mm}$, and a length of $200 \mathrm{~cm}$, was introduced into the common bile duct via an Olympus JFIT10 duodenoscope. The manometric catheter had three lateral openings of $0.5 \mathrm{~mm}$ in diameter at $2 \mathrm{~mm}$ intervals. The most distal end of the catheter was marked by six black rings $2 \mathrm{~mm}$ apart to facilitate positioning of the catheter in relation to the ampulla. Selective common bile duct cannulation was confirmed by aspiration of bile; fluoroscopy was not used.
The catheter was perfused continuously with sterile water using a low compliance pneumohydraulic capillary infusion pump (Arndorfer Medical Specialities, Wisconsin, USA) at a flow rate of $0.25 \mathrm{ml} / \mathrm{min}$. This was connected via a transducer to a computerised polygraph (Albyn Medical Version 6.0, UK). The transducer was calibrated before each study and catheter lumen occlusion produced a pressure rise in excess of $250 \mathrm{~mm} \mathrm{Hg} / \mathrm{s}$.

After recording duodenal pressure, which was taken as the zero reference, the papilla was cannulated and the catheter was withdrawn across the $\mathrm{SO}$ in $2 \mathrm{~mm}$ increments using the black marks on the catheter as a guide. Recordings were obtained for at least 60 seconds at each station. After two such pull-throughs, the catheter was repositioned so that all three channels recorded phasic SO 


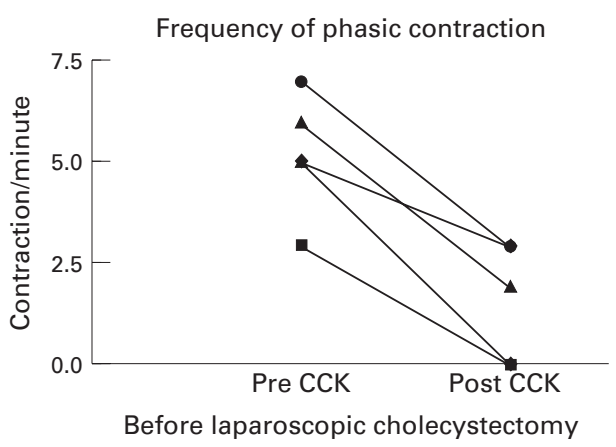

Amplitude of phasic contraction

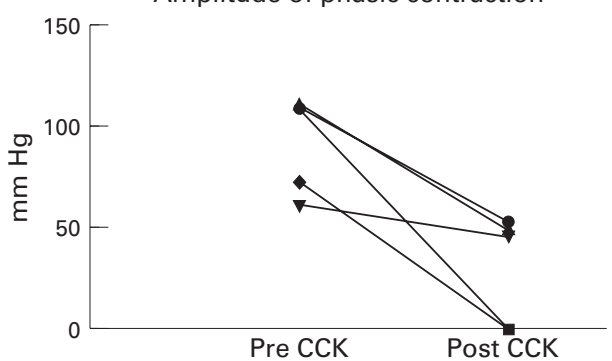

Before laparoscopic cholecystectomy

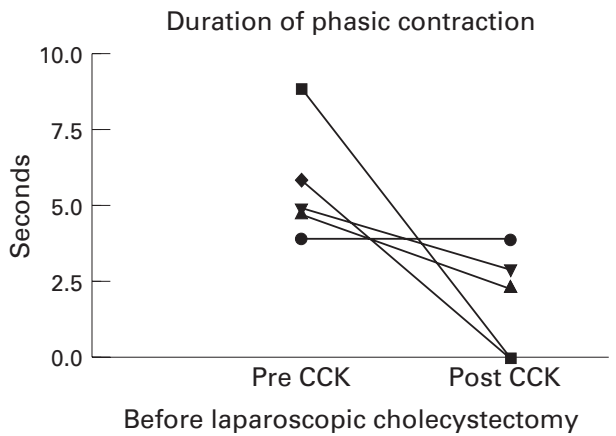

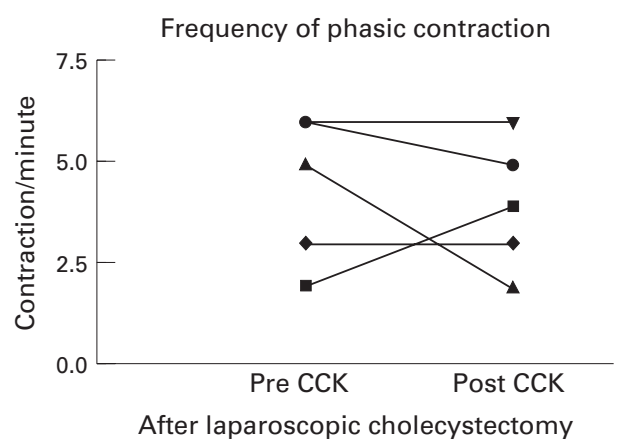

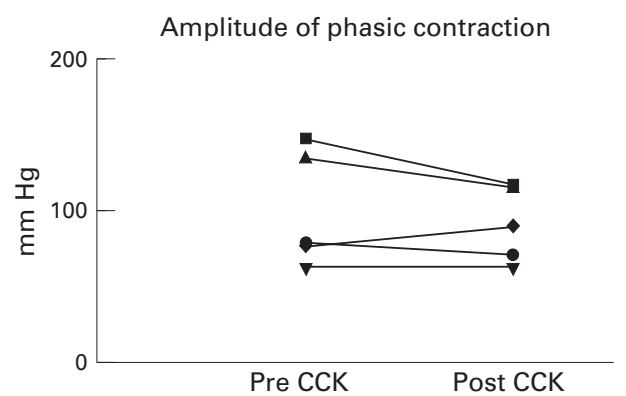

After laparoscopic cholecystectomy

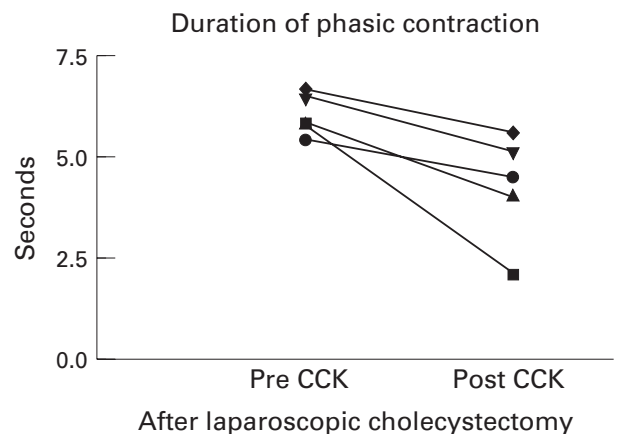

Figure 1: Variables of SO motor activity with CCK administration before and after laparoscopic cholecystectomy.

contractions. Cholecystokinin (Ferring Pharmaceuticals, Malmo, Sweden) was injected intravenously at $1 \mathrm{Ivy}$ Dog Unit/kg bodyweight over 60 seconds. After a 30 second equilibration period, recordings within the SO were obtained for two to three minutes. More prolonged recording proved impossible as CCK induced retching and discomfort. The total duration of recording was approximately 10 minutes.

ANALYSIS OF MANOMETRIC RECORDINGS

Manometric tracings were coded and analysed by an experienced observer (AP) who was blinded to the status of patients. Common bile duct pressure, basal SO pressure, and amplitude of the phasic contractions of the sphincter were determined with reference to the intraduodenal pressure. The mean amplitude of phasic contractions was calculated as the average of contractile amplitude minus basal pressure in each of the three channels. Average phasic contractile frequency was calculated as the number of phasic contractions divided by the duration of the recording period during which contractions were identified. The dura- tion of phasic contraction in seconds was measured from the onset of the major upstroke to the end of the wave. A motility index was calculated as the product of mean amplitude and frequency. ${ }^{4}$ Duodenal motor activity was monitored using a separate catheter which was taped to the outside of the endoscope.

\section{STATISTICAL ANALYSIS}

All data are reported as mean (1SE) unless otherwise stated. Paired Student's $t$ tests were used to compare variables before and after laparoscopic cholecystectomy. Baseline values - that is, values obtained before CCK administration, and those found after the administration of CCK injection were analysed separately. Differences with $p$ values of less than 0.05 were considered significant.

\section{Results}

All patients underwent uncomplicated laparoscopic cholecystectomy and completed questionnaires showed excellent symptomatic outcome in all cases. Biliary manometry was well tolerated and successful in all patients although 
TABLE 1 Sphincter of Oddi motility variables before and after laparoscopic cholecystectomy

\begin{tabular}{lll}
\hline Motility variable & $\begin{array}{l}\text { Before laparoscopic } \\
\text { cholecystectomy }\end{array}$ & $\begin{array}{l}\text { After laparoscopic } \\
\text { cholecystectomy }\end{array}$ \\
\hline $\begin{array}{l}\text { Mean CBD pressure (mm Hg) } \\
\text { Baseline }\end{array}$ & $5.5(3)$ & $6.8(2.3)$ \\
SO basal pressure (mm Hg) & $24(7)$ & $27(3)$ \\
SO phasic frequency (per minute) & $5.2(0.6)$ & $4.4(0.8)$ \\
SO phasic amplitude & $94(11)$ & $101(17)$ \\
SO phasic duration (seconds) & $5.7(0.8)$ & $6.1(0.2)$ \\
Motility index & $491(96)$ & $416(79)$ \\
After CCK & $22.6(6)$ & $26(4)$ \\
SO basal pressure (mm Hg) & $1.6(0.7)$ & $4(0.7)^{\star}$ \\
SO phasic frequency (per minute) & $30.5(12.5)$ & $94(11)^{\star}$ \\
SO phasic amplitude & $1.9(0.8)$ & $4.4(0.6)^{\star}$ \\
SO phasic duration (seconds) & $81(35)$ & $351(43)^{\star}$ \\
Motility index &
\end{tabular}

${ }^{\star} \mathrm{p}<0.05$ injection of CCK before and after laparoscopic cholecystectomy.

CBD, common bile duct.

one patient developed uncomplicated and rapidly resolving acute pancreatitis following her second manometric study.

Table 1 and fig 1 present the results of SO motor activity, and an example of manometric tracing is shown in fig 2 . There was a non-significant increase in mean common bile duct pressure after laparoscopic cholecystectomy. Baseline SO motility variables were very similar before and after laparoscopic cholecystectomy. Prior to laparoscopic cholecystectomy CCK injection consistently suppressed SO phasic activity. Mean basal SO pressure tended to fall after CCK but this did not achieve statistical significance. Thus, the major effect of CCK was that phasic contractions became significantly less frequent, were shorter in duration, and had lower amplitude than baseline values. Consequently, the motility index fell from 491 (96) to 81 (35). Following laparoscopic cholecystectomy CCK again did not significantly affect SO basal pressure. However, in notable contrast to the findings before surgery, CCK had only minimal effect on phasic contractions. Mean frequency and amplitude of phasic contractions were identical at baseline and after CCK, and although the mean duration of SO phasic contractions was reduced significantly by CCK, the magnitude of this effect was much less than that which occurred preoperatively. Indeed, the mean post-CCK phasic wave duration was signifi-

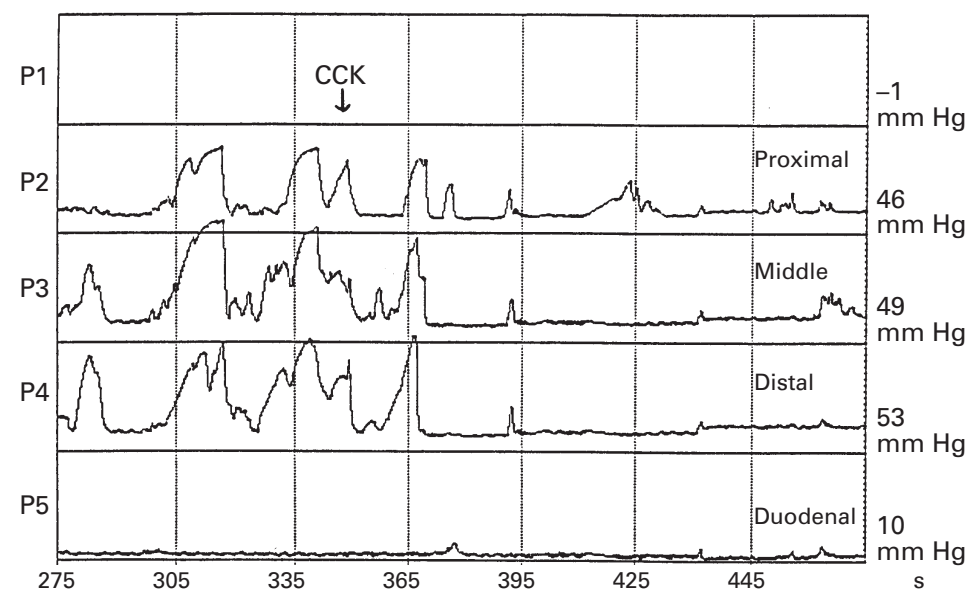

Figure 2: $\quad$ Manometric tracing of SO motility (the top three tracings represent proximal, middle, and distal sphincter pressure; the bottom tracing shows the duodenal pressure). cantly longer after cholecystectomy (table 1). The motility index fell with CCK injection from 416 (79) to 351 (43), and this relatively small change was significantly different from that measured preoperatively $(\mathrm{p}<0.01)$.

\section{Discussion}

SO motility was normal in the five subjects studied prior to laparoscopic cholecystectomy, and as anticipated, a pharmacological dose of CCK largely inhibited phasic SO activity and tended to reduce tonic pressure. This is consistent with findings from other studies using a similar perfusion technique in healthy volunteers. ${ }^{5-8}$ Following laparoscopic cholecystectomy there was a non-significant increase in mean common bile duct and tonic SO pressure, but the most striking finding was loss of inhibition of SO phasic activity following CCK injection.

This observation contrasts with the findings of other groups of reduced SO phasic contraction following CCK injection in the majority of patients investigated for post-cholecystectomy syndrome. ${ }^{679}$ The reasons for this discrepancy are not clear. Our observations relate to laparoscopic cholecystectomy rather than open cholecystectomy but it is unlikely that operative differences account for the divergent results. Our observations were obtained after only a relatively short postoperative period, while others have reported their findings in patients studied many years after cholecystectomy; it is therefore possible that our findings are a temporary, early phenomenon. Nevertheless, our findings of reduced responsiveness to CCK after cholecystectomy in humans are similar to those reported by Grace and Pitt in the cat. ${ }^{10}$ These investigators showed that while basal SO motility was unaffected by cholecystectomy, the sphincter was not relaxed by intraduodenal fat infusion or by an injection of CCK.

Our observations relate to pharmacological, rather than physiological doses of CCK and their clinical relevance is unclear. Plasma CCK concentrations were not measured, but the doses of CCK injected pre- and postoperatively were identical in each patient and differences in SO motility following cholecystectomy could not be due to differences in CCK plasma concentrations.

The actions of CCK on the gall bladder and SO are complex and are known to be mediated by myogenic and neuronal receptors. In the gall bladder, CCK stimulates postganglionic cholinergic neurones and this action is blocked by atropine. ${ }^{11} \mathrm{CCK}$ also directly stimulates gall bladder smooth muscle contraction as the atropine resistant effect is unaltered by complete denervation with tetrodotoxin. In contrast, CCK inhibits the SO by stimulating postganglionic non-adrenergic, non-cholinergic inhibitory neurones, and this action is inhibited by tetrodotoxin but not atropine. CCK also directly contracts the SO by stimulating excitatory receptors on the smooth muscle, and this action is not inhibited by tetrodotoxin. ${ }^{11}$

Under physiological conditions, the neurally mediated inhibitory effect of CCK on the SO 
overrides the direct excitatory effect of the hormone on the SO smooth muscle and the SO displays net relaxation following CCK injection. The mechanism of reduced inhibitory SO phasic activity to CCK following laparoscopic cholecystectomy observed in this study is unexplained. It is, however, possible that cholecystectomy, by dividing the nerves connecting the gall bladder and SO, results in altered responsiveness to CCK. This is analogous to the experimental observation of truncal vagotomy in the prairie dog which leads to an increase in baseline resistance to bile flow through the $\mathrm{SO}^{12}$ and an increase in frequency and amplitude of phasic SO contractions. ${ }^{13}$ This is also compatible with the finding that an injection of tetrodotoxin or lignocaine into the cystic duct of animals prevents SO relaxation in response to gall bladder distension. ${ }^{12}{ }^{14}$ It is therefore likely that gall bladder and SO function are coordinated by nerve fibres which pass from the gall bladder to the SO; such fibres have actually been demonstrated by anatomical dissection. ${ }^{2} 315$

Elevated SO basal pressure, ${ }^{79}{ }^{16}$ retrograde phasic wave contraction, ${ }^{6}$ and increase in phasic wave greater than seven contractions per minute (tachyoddia) ${ }^{617}$ are several of the manometric abnormalities described for SO dysfunction in patients with post-cholecystectomy syndrome. Some patients with SO dysfunction also exhibit paradoxical increases in basal pressure and phasic activity after CCK. ${ }^{18}{ }^{19}$ The subjects reported in this study were all asymptomatic at the time of repeat manometry and none displayed these manometric abnormalities. It may, therefore, be inappropriate to extrapolate from this observation that denervation of $\mathrm{SO}$ is the cause of $\mathrm{SO}$ dysfunction in patients with post-cholecystectomy syndrome.

The findings reported in this study may alternatively be explained by the possibility that the $\mathrm{SO}$ relaxes in response to large surges of bile flow. Gall bladder contraction results in enormous increases in bile flow into the common bile duct and it is possible that ductal distension directly inhibits SO function by local reflexes. Bile flows continuously through the SO following cholecystectomy and therefore SO relaxation does not occur following CCK injection due to a lack of common bile duct distension.
We have demonstrated that cholecystectomy is associated with altered SO motility with pharmacological doses of CCK. Their clinical relevance is unclear and their relation to post-cholecystectomy syndrome is unknown. Further studies are needed to clarify our observations.

1 Thune A, Saccone G, Scicchitano JP, Toouli J. Distension of the gallbladder inhibits sphincter of Oddi motility. Gut 1991; 32: 690-3.

2 Thune A, Jivegard L, Conradi N, Svanvik J. Cholecystectomy in the cat damages pericholedochal nerves and impairs reflex regulation of the sphincter of Oddi. Acta Chir Scand Suppl 1988; 154: 191-4.

3 Padbury RTA, Furness JB, Baker RA, Toouli J, Messenger JP. Projections of nerve cells from the duodenum to the JP. Projections of nerve cells from the duodenum to the
sphincter of Oddi and gallbladder of the Australian sphincter of Oddi and gallbladder of the
possum. Gastroenterology 1993; 104: 130-6.

possum. Gastroenterology 1993; 104: 130-6.
4 Kaufman HS, Shermak MA, May CA, Pitt HA, Lillemoe Kaufman HS, Shermak MA, May CA, Pitt HA, Lillemoe
KD. Nitric oxide inhibits resting sphincter of Oddi activity. Am f Surg 1995; 165: 74-80.

5 Sung JY, Chung SCS. Endoscopic stenting for palliation of malignant biliary obstruction. Dig Dis Sci 1995; 40: $1167-$ 73.

6 Toouli J, Roberts-Thompson IC, Dent J, Lee J. Manometric disorders in patients with suspected sphincter of Oddi dysfunction. Gastroenterology 1985; 88: 1243-50.

7 Geenen JE, Hogan WJ, Dodds WJ, Toouli J, Venu RP. The efficacy of endoscopic sphincterotomy after cholecystectomy in patients with sphincter of Oddi dysfunction. $N$ Engl f Med 1989; 320: 320-7.

8 Guelrud M, Mendoza S, Rossiter G, Villegas MI. Sphincter of Oddi in healthy volunteers. Dig Dis Sci 1990; 35: 38-46.

9 Toouli J, Geenen JE, Hogan WJ, Dodds WJ, Arndorfer RC. Sphincter of Oddi motor activity: a comparison between Sphincter of Oddi motor activity: a comparison between patients with common bile duct
Gastroenterology 1982;82: 111-7.

10 Grace PA, Pitt HA. Cholecystectomy alters the hormonal response of the sphincter of Oddi. Surgery 1987; 102: 18693.

11 Behar J, Biancani P. Effect of cholecystokinin and the octapeptide of cholecystokinin on the feline sphincter of Oddi and gallbladder. F Clin Invest 1980; 66: 1231.

12 Pitt HA, Roslyn JJ, Kuchenbecker SL, Doty JE, Denbesten $\mathrm{L}$. The role of cystic duct resistance in the pathogenesis of cholesterol gallstones. F Surg Res 1981; 30: 508 .

13 Pitt HA, Doty JE, Denbesten L, Kuckenbecker SL. Altered sphincter of Oddi phasic activity following truncal vagotomy. I Surg Res 1982; 32: 589-607.

14 Thune A, Thornell E, Svanvik J. Reflex regulation of flow resistance in the feline sphincter of Oddi by hydrostatic resistance in the feline sphincter of Oddi by hydrostatic pressure

15 Toouli J. The functional integration of gallbladder and sphincter of Oddi motor activity in humans. In: Hogan WJ, ed. The sphincter of Oddi primer for the pancreaticobiliary endoscopists. Philadelphia: Saunders, 1993: 133-42.

16 Neoptolemos JP, Bailey IS, Carr-Locke DL. Sphincter of Oddi dysfunction: results of treatment by endoscopic sphincterotomy. Br F Surg 1988; 75: 454-9.

17 Hogan WJ, Geenen JE, Venu RP, Dodds WJ, Helm J, Toouli J. Abnormally rapid phasic wave contractions of the human sphincter of Oddi (tachyoddia). Gastroenterology 1983; 84: 1189.

18 Rolny P, Arleback A, Funch-Jensen P, Kruse A, Ravnsbaeck J, Jarnerot G. Paradoxical response of sphincter of Oddi to IV injection of cholecystokinin or ceruletide. Manometric findings and results of treatment in biliary dyskinesia. Gut 1986; 27: 1507-11.

19 Hogan WJ, Geenen J, Dodds WJ, Toouli J, Venu R, Helm J. Paradoxical motor response to cholecystokinin (CCK-OP) in patients with suspected sphincter of Oddi dysfunction [abstract]. Gastroenterology 1982; 82: 1085. 